\title{
Teucrium chlorocephalum Čelak. (= Teucrium reverchonii Willk.), un endemismo andaluz poco conocido: Distribución y conservación
}

Federico Casimiro-Soriguer Solanas ${ }^{1} \&$ Juan J. Cebrián de la Serna ${ }^{2}$

'Área de Botánica (Dpto. de Botánica y Fisiología Vegetal). Facultad de Ciencias. Universidad de Málaga. Málaga.

${ }^{2}$ Ayuntamiento de Málaga. Málaga

\section{Correspondencia}

F. Casimiro-Soriguer

e-mail: fedeque@hotmail.com

Recibido: 2 septiembre 2020

Aceptado: 30 septiembre 2020

Publicado on-line: 11 noviembre 2020

Editado por: Teresa Navarro

\begin{abstract}
Resumen
Teucrium chlorocephalum Čelak. (= Teucrium reverchonii Willk.), es un endemismo andaluz reiteradamente confundido con Teucrium haenseleri Boiss. En este trabajo se actualiza la distribución conocida de esta especie en base a pliegos de herbario, se dan a conocer sus únicas poblaciones actualmente localizadas y se evalúa su estado de conservación.
\end{abstract}

Palabras clave: Teucrium, endemismo, Península Ibérica, Málaga, conservación.

\begin{abstract}
Teucrium chlorocephalum Čelak. (= T. reverchonii Willk.), a poor known andalusian endemism: Distribution and conservation.

Teucrium chlorocephalum Čelak. (= T. reverchonii Willk.), is an Andalusian endemism repeatedly confused with $T$. haenseleri Boiss. In this work, the known distribution of this species is updated based on herbarium vouchers, its only currently known populations are disclosed, and its conservation status is evaluated.
\end{abstract}

Key words: Teucrium, endemism, Iberian Peninsula, Málaga, conservation.
Teucrium chlorocephalum Čelak. (Fig. 1), es un caméfito cuyo nombre tradicionalmente aceptado en las floras básicas que abarcan el sur de la Península Ibérica: Flora Vascular de Andalucía Occidental, Flora Iberica y Flora Vascular de Andalucía Oriental, así como en la última monografía sobre la flora vascular andaluza (Cueto et al., 2018) es Teucrium reverchonii Willk. En World Checklist of Selected Plant Families (Govaerts et al., 2020) aparece T. chlorocephalum como nombre legítimo de $T$. reverchonii. Aunque la nomenclatura y sinonimias de $T$. chlorocephalum ya vienen recogidas en WCSP, nos parece necesario incluirlas aquí junto con sus tipos nomenclaturales y respectivas localidades tipo:

\section{Teucrium chlorocephalum Čelak. in Bot. Centralbl. 4(14): 186 (1883)}

( $\equiv T$. aragonese Hut., Porta et Rigo in Scheda! nec Loscos). Ind. loc.: "Provinc. Malacitana ad pagum de Alora, 1879". Typus: no designado.

= Teucrium haenseleri var. angustifolium Boiss., Voy. Bot. Espagne 2: 518 (1841), Ind. loc.: Varietas B in provinciâ Malacitanâ Haenseler. Lectotypus: GBOISS, G 00402108! (cf. Burdet et al. in Candollea 42(1): 121, 1987). (GBIF - Imagen digital).

= Teucrium reverchonii Willk. in Oesterr. Bot. Z. 41: 53 (1891), Ind. loc.: In regno Granatensi: Sierra de la
Pizarra in prov. Malacitana, in aridis. Reverchon (d. 11. Junii 1888). Lectotypus: "La Pizarra, in collibus squistosis aridis, Reverchon 1890", COI-WILLK 00047406 ! (cf. T. Navarro in Acta Botanica Malacitana 20: 173-265, 1995) (COI - Imagen digital).

$=$ Teucrium hispidulum Laza in Anales Jard. Bot. Madrid 6 (2): 335 (1946). Ind. loc.: Sierra Almijara, julio 1936. Typus: MA 98396!

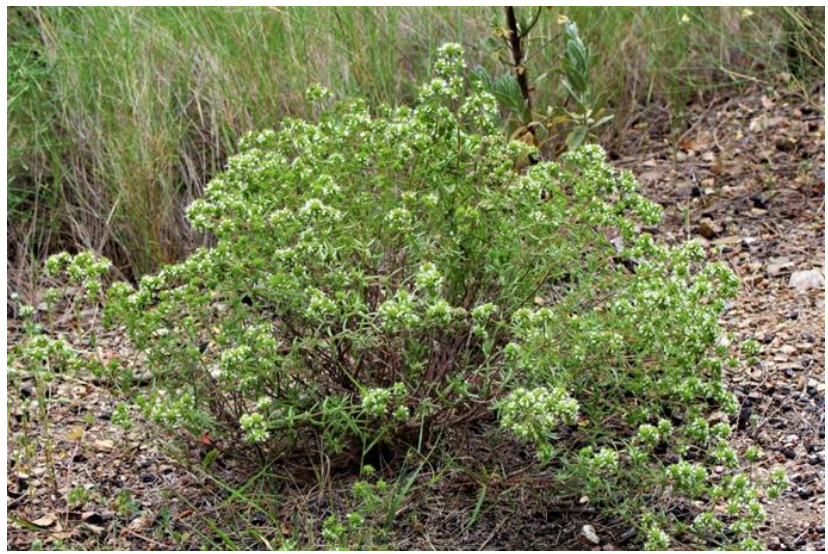

Figura 1. Teucrium chlorocephalum.

Figure 1. Teucrium chlorocephalum.

T. chlorocephalum es un endemismo del sur de la Península lbérica con una distribución limitada a la cordillera Bética Occidental, provincias de Cádiz, Málaga y Sevilla (Navarro, 2010; Devesa, 1986). 
Aunque es un taxón perfectamente definido ( $T$. reverchonii; Willkomm, 1891; Ruiz de la Torre, 1974; Devesa, 1986; Navarro, 2010; López González, 1980), una reiterada confusión con Teucrium haenseleri Boiss., ha propiciado una fuerte distorsión en el conocimiento sobre su área de distribución actual.

El ánimo principal de esta nota es actualizar los datos sobre la distribución actual de $T$. chlorocephalum en la Península lbérica, así como proponer una evaluación de amenaza según criterios UICN.

\section{Errores de interpretación}

$T$. reverchonii fue descrito de la Sierra de la Pizarra (Málaga; cf. Willkomm, 1891), sobre esquístos y substratos calcáreos. Las primeras citas erróneas que indican a este taxón asociado a substratos peridotíticos, confundiéndolo con $T$. haenseleri, son de Rivas Goday \& Rivas Martínez (1969), Rivas Goday (1973) y Rivas Martínez et al. (1973). Posteriormente, López González (1975), al estudiar la Sierra de Aguas, sierra peridotítica aneja a la Sierra de la Pizarra, identificó claramente a $T$. haenseleri como una planta ligada a las peridotitas, y a $T$. reverchonii como una planta que no crecía en estos substratos peridotíticos, sino en pizarras. Sin embargo, $T$. reverchonii fue posteriormente conside- rado de nuevo como un serpentinófito (Rivas Martínez, 1978), al confundirlo con T. haenseleri. Este error, que fue de nuevo claramente resuelto por López Gonzalez (1980), se retomó en las dos primeras décadas del siglo XXI, donde reiteradamente se citó a $T$. reverchonii como una planta ligada a los substratos peridotíticos, considerándolo un serpentinófito (Gavira \& Pérez Latorre, 2005; Pérez Latorre et al., 2012, 2013a, 2013b; Pérez Latorre \& Hidalgo Triana, 2017; Gavira et al., 2016).

Por último, se volvió a resolver esta confusión en Pérez Latorre et al. (2018), gracias a la revisión llevada a cabo por uno de los autores de la presente nota (FCS) del material de herbario identificado como $T$. reverchonii y $T$. haenseleri y depositado en el herbario MGC. En esa revisión se constató que todas las plantas recolectadas sobre peridotitas pertenecian a $T$. haenseleri. En este mismo sentido hemos visto material recolectado e identificado como $T$. reverchonii por Rivas Goday et al. en 1965, proveniente de la Sierra de Carratraca (VAL 160005), llamada también Sierra de Aguas, y como era de esperar, corresponde a $T$. haenseleri.

Un resumen de las diferencias morfológicas más significativas entre $T$. reverchonii y $T$. haenseleri se recogen en la Tabla 1 y Figura 2.

Tabla 1. Diferencias morfológicas entre T. chlorocephalum y T. haenseleri.

Table 1. Morphological differences between T. chlorocephalum and T. haenseleri.

\begin{tabular}{|c|c|c|c|}
\hline Taxón & Indumento del tallo & Indumento del cáliz & $\begin{array}{l}\text { Morfología de la } \\
\text { inflorescencia }\end{array}$ \\
\hline T. chlorocephalum & $\begin{array}{l}\text { Tallo con pelos simples y pelos } \\
\text { ramificados, generalmente } \\
\text { aplicados, a veces hirsutos, } \\
\text { mezclados con indumento } \\
\text { pubescente (Figura } 2-\mathrm{A} \text { ) }\end{array}$ & $\begin{array}{l}\text { Cáliz hirsuto en un 1/3-1/2 } \\
\text { basal, con dientes } \\
\text { enteramente glabros o } \\
\text { ciliados en los márgenes } \\
\text { (Figura 2-C) }\end{array}$ & $\begin{array}{l}\text { Inflorescencia con } \\
\text { cabezuelas esféricas (Figura } \\
2-E \text { ) }\end{array}$ \\
\hline T. haenseleri & $\begin{array}{l}\text { Tallos hirsutos con pelos } \\
\text { simples, patentes, no } \\
\text { glandulíferos, mezclados con } \\
\text { abundantes pelos glandulíferos } \\
\text { cortos } \\
\text { (Figura 2-B) }\end{array}$ & $\begin{array}{l}\text { Cáliz enteramente hirsuto, } \\
\text { con pelos eglandulíferos } \\
\text { largos mezclados con pelos } \\
\text { más cortos glandulíferos y } \\
\text { glándulas (Figura 2-D) }\end{array}$ & $\begin{array}{l}\text { Inflorescencia con } \\
\text { cabezuelas ovoides o más o } \\
\text { menos cónicas, raramente } \\
\text { esféricas (Figura } 2-\mathrm{F} \text { ) }\end{array}$ \\
\hline
\end{tabular}

\section{Distribución}

Las citas bibliográficas fiables más recientes que conocemos basadas en pliegos de herbario son de los alrededores de Coripe (Cádiz, Sevilla; cf. Devesa, 1986) y Sierra de la Pizarra (Málaga; cf. López González, 1980); en Flora Iberica, Navarro (2010) citó la especie en Málaga y con dudas en las provincias de Cádiz y Sevilla. En ANTHOS (2020), aparecen 2 localidades fuera de este ámbito geográfico, una de Puerto Real (Navarro, 1995) basada en un pliego testigo que en realidad se trata de Teucrium capitatum (MGC 39592) y otra en la Sierra de Gádor (Almería) basada en una cita de Boissier de $T$. haenseleri var. angustifolium, pero es un error de transcripción ya que Boissier en esa sierra solo citó a T. haenseleri s. str.

En la provincia de Málaga hay citas de $T$. reverchonii, de Parauta (Navarro, 1995) y Coín (Cabezudo et al., 1991), cuyos pliegos de referencia están en el herbario MGC, y que hemos podido comprobar que son erróneas.

En GBIF (2020) hemos podido revisar algunos pliegos recolectados en las sierras peridotíticas malagueñas identificados como $T$. reverchonii y depositados en $W$, que como era de esperar, corresponden a T. haenseleri (GBIF - imagen digital). Por último, tenemos que señalar que ANTHOS (2020) recoge la presencia en Sierra Bermeja de $T$. reverchonii, basada en las citas de López González 
(1980) y Boissier (1841). Estas citas son erróneas y se deben a errores de transcripción, ya que López González (1980) descartó la presencia de $T$. reverchonii sobre peridotitas, indicando expresa- mente que la especie citada en Sierra Bermeja era $T$. haenseleri y no $T$. reverchonii, y Boissier (1841) en esa sierra solo citó a $T$. haenseleri s. str.

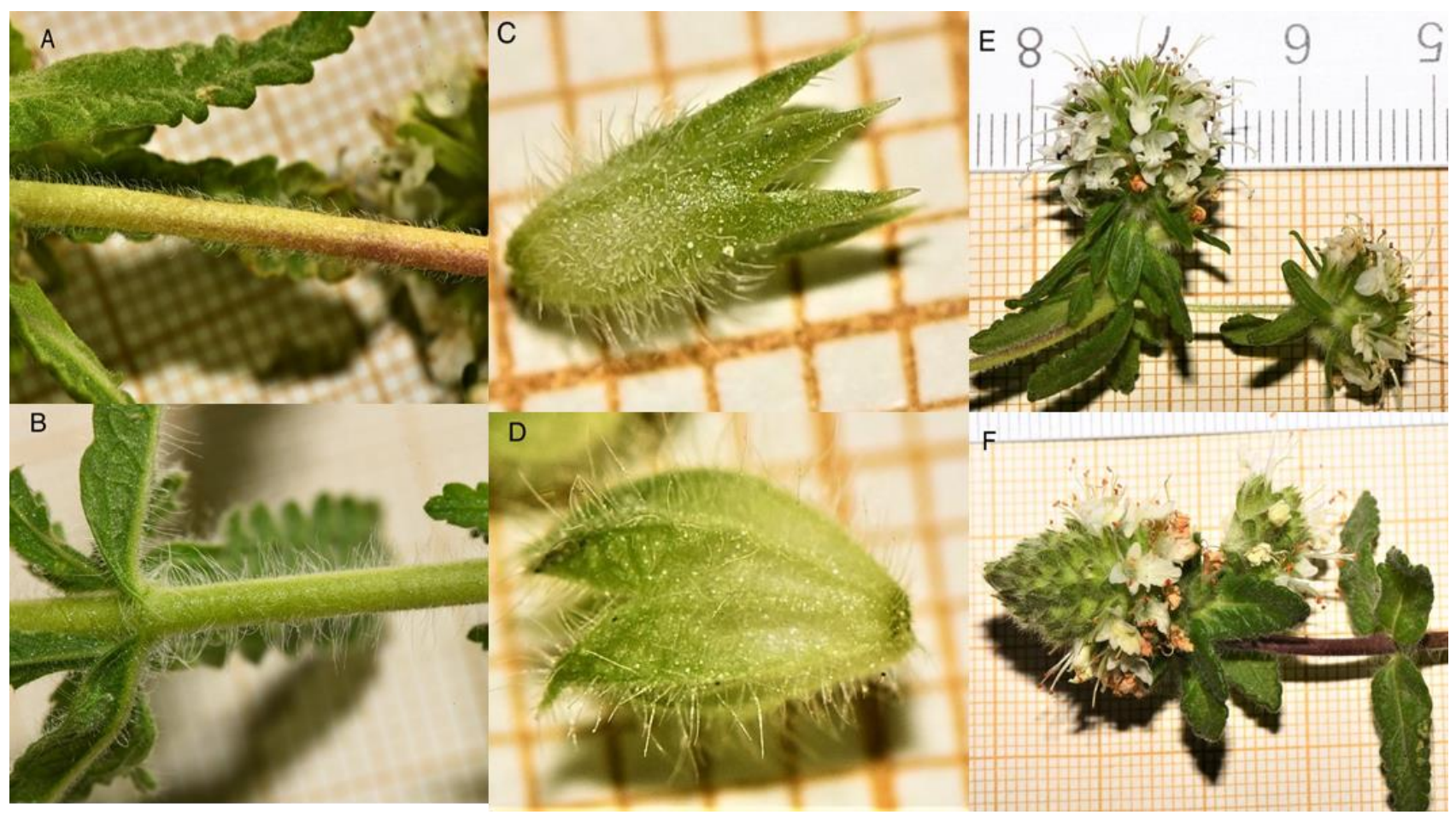

Figura 2. Diferencias morfológicas más significativas entre T. chlorocephalum y T. haenseleri.

T. chlorocephalum (MGC 93114): A. Indumento del tallo; C. Indumento del cáliz; E. Morfología de la inflorescencia. T. haenseleri: B. Indumento del tallo; D. Indumento del cáliz; F. Morfología de la inflorescencia.

Figure 2. Most significant morphological differences between T. chlorocephalum and T. haenseleri.

T. chlorocephalum (MGC 93114): A. Stem indumentum; C. Calyx indumentum; E. Inflorescence morphology. T. haenseleri (MGC 93116): B. Stem indumentum; D. Calyx indumentum; F. Inflorescence morphology

En 2005, uno de los autores de este trabajo (JCS) localizó una serie de núcleos poblacionales de $T$. chlorocephalum en el Parque Natural Montes de Málaga y en los aledaños de Málaga capital. Este hecho, junto con la revisión del material del herbario MGC mencionada anteriormente y de otros herbarios como SEV, GDA, MA, VAL, B, G, COI y W (acrónimos según Thiers, 2020), y las consultas bibliográficas realizadas, nos permiten hacer una buena aproximación a la distribución de $T$. chlorocephalum en la Península lbérica.

Las localidades conocidas de $T$. chlorocephalum en la Península Ibérica, respaldadas por pliego de herbario (sin incluir los pliegos de la localidad tipo de T. reverchonii, recolectados por É. Reverchon y distribuidos por los principales herbarios europeos, en la exsiccata "Plantes de I'Andalousie"), son las siguientes:

\section{Provincias de Cádiz - Sevilla}

Aunque desde su descripción $T$. chlorocephalum parecía un endemismo malagueño, Devesa (1986) citó esta especie (como $T$. reverchonii) en la zona comprendida entre Coripe y Olvera, área donde contactan las provincias de Cádiz y Sevilla. La última referencia de su presencia en la zona es un pliego recolectado en 1999. Recientemente se han dado por extintas estas poblaciones (Gutiérrez et al., 2019).

Cádiz: Entre Algodonales y Coripe, 3/6/1999, C. Santa-Bárbara (SEV 142534). Sevilla: Río Guadalporcún, 13/06/1978, Ruíz de Clavijo \& Silvestre (SEV 36238); Entre Coripe y Algodonales, 17/06/1977, Ruíz de Clavijo (SEV 28926).

\section{Provincia de Málaga}

\section{Sierra de la Pizarra}

Esta es la localidad clásica de $T$. reverchonii, y seguramente también de T. chlorocephalum, ya que esta sierra se localiza en los términos municipales de Ardales y Álora. La última referencia en dicha zona de la que tenemos constancia es de López Gonzalez (1980). En los últimos años se han buscado estas poblaciones, pero sin éxito (J. Fuentes, com. pers.).

Málaga: Sierra de la Pizarra, $30 S$ UF 4184, localidad clásica, $720 \mathrm{~m}$, pizarras básicas, 13/05/1979, $P$. Cantó, P. Cubas, G. López \& C. Pardo (MA 211990). 2. Parque Natural Montes de Málaga y alrededores de Málaga capital

Anteriormente a las actuales poblaciones halladas dentro del Parque Natural Montes de Málaga, todas las referencias previas que conocemos de esta zona 
se correspondían a pliegos de herbario recolectados en 1977 y 1988.

Málaga: Málaga, Pantano del Agujero, 15/5/1977, A. Asensi \& B. Díez (MGC 4074); Cerrado de Calderón, 25/5/1977, A. Asensi \& B. Díez (MGC 4077); Montes de Málaga, camino forestal de Las Contadoras a Jotrón, 700 msnm., 13/5/1988, A. Asensi \& B. Díez (MGC 24620); Parque Natural Montes de Málaga, próximo a la Fuente de la Reina, esquistos margosos, 05/06/2016, G. Blanca, J. Fuentes \& M. Cueto (GDA 63392); P. N. Montes de Málaga, Cencerrilla, 18/05/2020, Juan J. Cebrián (MGC 93113); P. N. Montes de Málaga, Mirador Martínez Falero, 18/05/2020, Juan J. Cebrián (MGC 93114); P. N. Montes de Málaga, cerca del área recreativa $\mathrm{El}$ Cerrado, 09/06/2020, Juan J. Cebrián (MGC 93115).

\section{Mijas Costa.}

De esta localidad, en las proximidades de la Punta de Calaburra, solo tenemos constancia por un pliego de herbario identificado originalmente como $T$. haenseleri. Área que en la actualidad está casi totalmente urbanizada.

Málaga: Mijas, Calaburra, El Chaparral, 20 msnm., jaral, esquistos, 1/4/1992, J.M. Nieto \& Pérez Latorre (MGC 35558).

4. Montes de Almogía.

De esta zona, tenemos constancia por dos pliegos, uno de ellos identificado originalmente como $T$. haenseleri. Creemos también que a esta localidad se refiere la asociación Saturejo malacitanae Corydothymetum capitati var. de Teucrium haenseleri (Pérez Latorre et al., 2008: 235), descrita de los Montes de Almogía y respaldada por el pliego antes mencionado.

Málaga: Almogía, cerca del pueblo, matorral, calizas, 15/5/2005, Pérez Latorre (MGC 61731); La Aljaima, esquistos, $100 \mathrm{msnm}, 05 / 06 / 1974$, 30SUF 529/675, Ruiz de la Torre (MA 835028).

Tabla 2. Poblaciones de Teucrium chlorocephalum, localidades y número de individuos.

Table 2. Populations of $\mathrm{T}$. chlorocephalum, location and number of specimens.

\begin{tabular}{lllll}
\hline Población & Localidad & UTM & Altitud (msnm) & № de individuos \\
\hline 1 & P.N. Montes de Málaga & 30SUF7545273836 & 585 & 23 \\
2 & P.N. Montes de Málaga & $30 S U F 7635374226$ & 750 & 13 \\
3 & P.N. Montes de Málaga & $30 S U F 7648374998$ & 650 & 11 \\
4 & P.N. Montes de Málaga & 30SUF7551474288 & 560 & extinta \\
5 & Cerrado de Calderón & 30SUF76856633 & 175 & 1 \\
\hline
\end{tabular}

\section{Sierra Almijara.}

La única referencia que tenemos de la presencia de la especie es la que indicó Laza Palacios (1946) cuando describió su T. hispidulum. Esta localidad es, por su hábitat, distinta al resto ya que el mencionado autor la citó en colinas a 1400 msnm., en el sotobosque de pinares de $P$. pinaster.

Málaga: Sierra Almijara, 07/1936, M. Laza Palacios (MA 98396)

\section{Sierra de Alcaparaín}

De esta sierra próxima a la Sierra de la Pizarra, tenemos constancia de la presencia de la especie, solo por un pliego recolectado y correctamente identificado por C. Vicioso en 1930.

Málaga: Carratraca, Sierra de Caparaín, 20/06/1930, C. Vicioso (VAL 155432).

\section{Hábitat}

Con los datos disponibles, se trata de una especie termófila, ocupando un rango altitudinal aproximado de 0 a 800 msnm., en matorrales más o menos abiertos y taludes, asentados tanto en calizas como esquistos. En las únicas poblaciones que conocemos tiene un claro carácter colonizador y termófilo, sobre todo en taludes con matorrales poco desarrollados en exposiciones soleadas.

\section{Conservación}

T. chlorocephalum no está recogido en ninguno de los catálogos de flora amenazada española ni andaluza. Sin embargo, en un reciente trabajo sobre los taxones más amenazado de la Flora Vascular de Andalucía Oriental (Gutiérrez et al., 2019), aparece entre los 48 taxones más amenazados de la misma (como T. reverchonii).

Con la información disponible sobre la distribución histórica de $T$. chlorocephalum y los trabajos de localización y censos realizados por uno de los autores de este trabajo (JCS) de sus únicas poblaciones actualmente conocidas, se propone a $T$. chlorocepalum como una especie amenazada, con la categoría UICN de En Peligro, EN (B1a, D).

Aunque somos conscientes de que un mayor esfuerzo de muestreo, en las localidades confirmadas por anteriores recolecciones, seguramente permitiría encontrar más poblaciones de esta especie, no es menos cierto que todas esas localidades fueron herborizadas hace al menos 15 años y la mayoría hace más de 20 años, sin que hayan vuelto a ser encontradas. 
A día de hoy solo están confirmadas la existencia de 5 poblaciones (Tabla 2), una de ellas seguramente desaparecida a causa de labores forestales, todas ellas en el término municipal de Málaga capital.

\section{Agradecimientos}

Nuestro agradecimiento a Baltasar Cabezudo, a Pedro Fructuoso Gallardo, a José M. López Moreno y a Victoria Robles Mata. En especial a José García Sánchez, conservador del Herbario de la Universidad de Málaga por habernos atendido con la máxima eficiencia durante el confinamiento por COVID-19, y a Rafael A. Haro Ramos, Director del Parque Natural de los Montes de Málaga por la atención prestada en idénticas circunstancias. A Sara Casimiro-Soriguer por su traducción del alemán del protólogo de $T$. chlorocephalum, y a J. Fuentes por informarnos de la búsqueda de este Teucrium en la Sierra de la Pizarra.

\section{Bibliografía}

ANTHOS (2020). Sistema de información de las plantas de España. Real Jardín Botánico, CSIC Fundación Biodiversidad.

Boissier, P. E. (1839-1845). Voyage botanique dans le Midi de L'Espagne pendant l'Année 1838, vol. 2. París.

Cabezudo, B., Nieto Caldera, J.M., Navarro, T. (1991). Cátalogo de las labiadas (Labiatae) malacitanas (Málaga, España). Acta Botanica Malacitana, 16 (2), 347-371.

Čelakovský, L. (1883). Ueber einige Arten der Gattung Teucrium. Botanisches Zentralblatt, 14, 186-188.

Cueto, M., Melendo, M., Giménez, E., Fuentes, J., López, E., Blanca, G. (2018). First updated checklist of the vascular flora of Andalusia (S of Spain), one of the main biodiversity centres in the Mediterranean Basin. Phytotaxa, 339, 1-95.

Devesa, J.A. (1986). Teucrium haenseleri Boiss., T. reverchonii Willk. y $T$. algarbiense (Coutinho) Coutinho en Andalucía occidental. Lagascalia, 14 (1), 85-87.

Gavira, O. \& Pérez Latorre, A.V. (2005). Aportaciones al catálogo florístico del Valle del Genal. Acta Botanica Malacitana, 30, 197-209.

Gavira-Romero, O., Hevilla-Ordoñez, S. \& CanoGarcía, E. (2016). Aportaciones a la flora de Sierra Alpujata y Sierra Blanca (Coín, Málaga). Botanica Complutensis, 40, 75-102

GBIF (2020). Global Biodiversity Information Facilities. www. GBIF. org.

Govaerts RA, Paton A, Harvey Y, Navarro T, Del Rosario Garcia Pena M (2020). World Checklist of Lamiaceae. Facilitated by the Royal Botanic Gardens, Kew. Published on the Internet; http://wcsp.science.kew.org/ September 2020.

Gutiérrez, L., Fuentes, J., Cueto, M. \& Blanca, G. (2019). Top ten de las plantas más amenazadas de Andalucía Oriental: taxones endémicos y no endémicos. Acta Botanica Malacitana, 44, 5-33.
Laza Palacios, M. (1946). Estudios sobre la flora y la vegetación de las Sierras Tejeda y Almijara. Boletín Instituto Botánico Cavanilles 6 (2): 217370.

López González, G. (1975). Contribución al estudio florístico y fitosociológico de la Sierra de Aguas. Acta Botanica Malacitana, 1, 81-205.

López González, G. (1980). Notas críticas a la flora malacitana. Anales Jardín Botánico Madrid, 36, 275-289.

Navarro, T. (1995). Revisión del género Teucrium L., sección Polium (Mill.) Schreb., en la Península Ibérica y Baleares. Acta Botanica Malacitana, 20, 173-265.

Navarro, T. (2010). Teucrium L. In R. Morales, A. Quintanar, F. Cabezas, A.J. Pujadas \& S. Cirujano -eds.- Flora Iberica: Verbenaceae-LabiataeCallitrichaceae, vol.12 (pp. 30-166). Real Jardín Botánico. CSIC. Madrid.

Pérez-Latorre A.V., Caballero, G., Casimiro-Soriguer, F., Gavira, O. \& Cabezudo, B. (2008). Vegetación del sector Malacitano-Axarquiense (Comarca de la Axarquía, Montes de Málaga y Corredor de Colmenar). Málaga (España). Acta Botanica Malacitana, 33, 215-270.

Pérez-Latorre A.V., Casimiro-Soriguer, F., Gavira, O. \& Cabezudo, B. (2012). Vegetación de la Reserva de la Biosfera Sierra de las Nieves: Río Grande y Sierras Prieta y Blanquilla (Málaga, España). Acta Botanica Malacitana, 37, 103-140.

Pérez-Latorre, A.V., Hidalgo-Triana, N., CasimiroSoriguer, F. \& Cabezudo, B. (2013). Flora y vegetación serpentinícola Ibérica: Sierra de Alpujata y de la Robla (Málaga, España). Lagascalia, 33, 43-74.

Pérez-Latorre, A.V., Hidalgo-Triana, N. \& Cabezudo, B. (2013). Composition, ecology and conservation of the south-lberian serpentine flora in the context of the Mediterranean basin. Anales Jardín Botánico Madrid, 70(1), 62-71.

Pérez-Latorre, A.V. \& Hidalgo-Triana, N. (2017). Los serpentinófitos suribéricos. Las joyas botánicas de Sierra Bermeja. Revista Takurunna, 6-7, 111112.

Pérez-Latorre, A.V., Hidalgo-Triana, N. \& Cabezudo, B. (2018). New data on the southern Iberian serpentinophyte flora (Andalusia, Spain). Mediterranean Botany, 39 (2), 151-155.

Rivas Goday, S. \& S. Rivas-Martínez (1969). Matorrales y tomillares de la Península Ibérica comprendidos en la clase Ononido-Rosmarinetea. Anales Instituto Botánico Cavanilles, 25, 7-201.

Rivas-Goday, S. (1973). Plantas serpentinícolas y dolomitícolas del Sur de España. Boletín Sociedad Broteriana, 47 (24 serie), 161-178.

Rivas Martínez, S., Izco, J. \& Costa, M. 1973. Asplenium cuneifolium Viv. (A. serpentini Tausch.) en Sierra Bermeja (Málaga). Trabajos Departamento Botánica y Fisiología Vegetal, 6, 23-30.

Rivas Martínez, S. (1978). De plantis hispaniae notulae systematicae, chorologicae et ecologicae 
III. Anales Instituto Botánico Cavanilles, 34 (2), 539-552.

Ruíz de la Torre, J. (1974). Teucria malagueños de hojas verticiladas. Boletín Estación Central de Ecología, 3(5), 83-95.

Thiers, B. (2020). Index Herbariorum: A global directory of public herbaria and associated staff.
New York Botanical Garden's Virtual Herbarium. http://sweetgum.nybg.org/ih/.

Willkomm, M. (1891). Ueber neue und kritische Pflanzen der spanisch-portugiesischen und balearischen Flora. Oesterreichische botanische Zeitschrift, 41(1), 51-54. 